\title{
VI Länderkompetenz für Mietenregulierung?
}

Neben diesen erheblichen materiell-rechtlichen Bedenken stellt sich aber die Frage, ob die Länder überhaupt neben den mietregulatorischen Interventionen des Bundes ergänzende Regelungen erlassen dürfen.

Ausgangspunkt aller Diskussionen ist die verfassungsrechtliche Regel, dass grundsätzlich den Ländern nach Art. 70 GG die Gesetzgebungskompetenz zukommt. Es gilt daher auch grundsätzlich eine Vermutungswirkung für die Kompetenz der Länder zur Gesetzgebung. ${ }^{109}$ Das Grundgesetz geht damit von einem „Regel-Ausnahme-Verhältnis zu Gunsten der Länder“110 aus. Daher muss - in der Diktion des BVerfG - gleichsam „der "Nachweis‘ geführt werden“111, dass eine Bundeskompetenz vorliegt. Es muss im Fall des Vorliegens einer konkurrierenden Gesetzgebungskompetenz des Bundes ferner gezeigt werden, dass sich das Land mit der angegriffenen Regelung zu einer auf dieser Kompetenz beruhenden abschließenden Regelung in verfassungswidriger Weise in Widerspruch setzt.

Diese Frage ist vorliegend schrittweise zu untersuchen. So ist zunächst im Ausgangspunkt festzustellen, dass Mietpreisregelungen - insoweit unstreitig jedenfalls auch unter die Kompetenzmaterie „bürgerliches Recht“ des Bundesgesetzgebers gemäß Art. 72, 74 Abs. 1 Nr. 1 GG fallen (dazu 1.). Fraglich ist sodann, ob der Bund tatsächlich abschließend von dieser konkurrierenden Gesetzgebungskompetenz Gebrauch gemacht hat (dazu 2.). Selbst wenn sodann den Ländern nach Art. 70 GG weiterhin eine Kompetenz für das Wohnungswesen zukäme, die auch ein Mietpreisrecht umfasste (dazu 3.), würde die abschließende Ausübung der Bundeskompetenz eine Regelung der Länder jedenfalls dann unterbinden, wenn diese eine kompetenz- und treuekonforme Regelung des Bundes in treuwidriger Weise unterläuft bzw. konterkariert, also nicht bloß abweichend, sondern widersprüchlich ist. Ein solcher widersprüchlicher Regelungsgehalt ist vorliegend in Bezug auf die einzelnen Kernbestimmungen des Gesetzes und die Gesamtregelung sorgfältig zu prüfen (dazu 4.). Vor diesem Hintergrund stellt sich sodann die Frage, ob aus dem Gedanken einer Länderkompetenz für das „Wohnungswesen“ in Kombination mit wechselseitigen Rücksichtnahmepflichten von Bund und Ländern aus dem Grundsatz der Bundes- bzw. Landestreue eine Re-

109 Zutreffend erinnert an diesen Ausgangspunkt Kingreen Zur Gesetzgebungskompetenz der Länder für das Öffentliche Mietpreisrecht bei Wohnraum, 2020, Kurzgutachten für die Bundestagsfraktion DIE LINKE, S. 6, abrufbar im WWW unter der URL https://www.linksfraktion.de/fi leadmin/user_upload/PDF_Dokumente/2020/200218_Kingreen_Mietendeckel_Kompetenz.pdf (zuletzt abgerufen am 1.7.2020).

110 BVerfGE 111, 226, Rn. 79 - juris; siehe dazu wiederum zutreffend Kingreen aaO, S. 6.

111 BVerfGE 42, 20, Rn. 41 - juris; wiederum zu Recht hervorgehoben bei Kingreen aaO, S. 6.

Ә OpenAccess. (C) 2020 Juergen Kuehling, publiziert von De Gruyter. (cc) BY-NC-ND Dieses Werk ist lizenziert unter der Creative Commons Attribution-NonCommercial-NoDerivatives 4.0 License.

https://doi.org/10.1515/9783110719031-006 
gelungskompetenz der Länder für eine Mietpreisregulierung folgt, selbst wenn diese in Konflikt gerät mit dem Gestaltungswillen des Bundesgesetzgebers (da$\mathrm{zu}$ 5.).

\section{Mietpreisrecht als Element der konkurrierenden Gesetzgebungskompetenz des Bundes nach Art. 72, 74 Abs. 1 Nr. 1 GG}

Nach Art. 72, 74 Abs. 1 Nr. 1 GG kommt dem Bund die konkurrierende Gesetzgebungskompetenz für das Mietrecht als Teil des „bürgerlichen Rechts“ zu. Bestandteil des Mietrechts ist das sogenannte „soziale“ Mietrecht, ${ }^{112}$ wozu das „Mietpreisrecht“ gehört. ${ }^{113}$ Vor diesem Hintergrund hat der Bundesgesetzgeber sämtliche Bestimmungen der Preisbindungen im Mietrecht und zuletzt auch die Regelungen zur sogenannten „Mietpreisbremse“ auf diesen Kompetenztitel gestützt. ${ }^{114}$ Dabei hatte der Gesetzgeber die „Mietpreisbremse“ gerade auch mit dem Ziel der Bekämpfung einer Gentrifizierung und dem Schutz einkommensschwacher Haushalte vor Verdrängung begründet, worauf das BVerfG in seiner materiell-rechtlichen Prüfung explizit abgestellt hatte. ${ }^{115}$ Das BVerfG hatte bei seiner Prüfung des Gesetzes zwar keine explizite kompetenzrechtliche Prüfung vorgenommen, aber offensichtlich deshalb, weil keinerlei kompetenzrechtliche Bedenken vorlagen, auch nicht hinsichtlich der Ausgestaltung der verbliebenen Konkretisierungsspielräume für die Länder. Kompetenzrechtlich hat das BVerfG die Konditionierung der Möglichkeiten der Länder, die Miethöhe zu regulieren, durch den Bund einschließlich der prozeduralen Anforderungen an die Begründung entsprechender Landesrechtsverordnungen sogar begrüßt und Letztere als Baustein für die Verfassungskonformität des Gesamtregulierungsansatzes herangezogen. ${ }^{116}$ Auch insoweit sind keine kompetenzrechtlichen Bedenken aufge-

112 Degenhart in: Sachs, GG, Kommentar, 8. Aufl. 2018, Art. 74 Rn. 81; Sannwald in: SchmidtBleibtreu/Hofmann/Henneke, GG, Kommentar, 14. Aufl. 2018, Art. 74 Rn. 232.

113 Oeter in: v. Mangoldt/Klein/Starck, GG, Kommentar, 7. Aufl. 2018, Art. 74 Rn. 10; Schede/ Schuldt NVwZ 2019, 1572 (1574); Wissenschaftlicher Dienst des Deutschen Bundestages, Gesetzliche Mietpreisregulierung durch die Länder aufgrund der Gesetzgebungskompetenz für das Wohnungswesen, WD 3-3000-029/19 vom 5.2.2019, S. 3; Wolfers/Opper DVBl. 2019, 1446 (1447f.). 114 BT-Drs. 18/3121, S. 19.

115 BVerfG, Beschl.v. 18.7.2019-1 BvL 1/18, Rn. 72, abrufbar im WWW unter der URL http://www. bverfg.de/e/lk20190718_1bvl000118.html - ECLI:DE:BVerfG:2019:1k20190718.1bvl000118 (zuletzt abgerufen am 1.7.2020).

116 BVerfG, aaO, Rn. 113. 
worfen worden. Insofern ist unstrittig davon auszugehen, dass der Bund über eine konkurrierende Gesetzgebungskompetenz zur Mietpreisregulierung einschließlich einer Kompetenz zur Konditionierung der Konkretisierungs- und Anwendungsmöglichkeiten der Länder verfügt und diese treuekonform durch die Ausgestaltung im BGB genutzt hat (dazu sogleich 2.).

\section{Reichweite des Gebrauchmachens dieser Kompetenz im BGB}

Das BGB trifft wie bereits oben dargestellt (III.1.) umfassende Regelungen zum Mietpreisrecht und insbesondere zu den Erhöhungsmöglichkeiten für Bestandsmieten ebenso wie zur zulässigen Miethöhe bei Neuvermietungen. Es stellt sich die Frage, ob diese Bestimmungen abschließend sind. Dies ist hier in Ausnahme des allgemeinen Grundsatzes (dazu a)) aufgrund der klaren Ergebnisse der systematischen, teleologischen und genetischen Auslegung (dazu b)) anzunehmen. Es wird auch deutlich, dass eine Beschränkung der landesrechtlichen Gestaltungsmöglichkeiten vom Bundesgesetzgeber gerade gewollt war (dazu c)). Aus der Rechtsprechung des BVerfG und insbesondere aus dem Beschluss vom 23. März 1965 lassen sich auch keine abweichenden Erkenntnisse ableiten (dazu d)).

\section{a) Ausgangspunkt: im Zweifel nicht abschließend}

Die Frage, ob es sich bei diesen bundesgesetzlichen Regelungen um abschließende Bestimmungen handelt, ist, sofern das Gesetz dies nicht explizit festlegt, im Rahmen einer Interpretation der Vorschriften $\mathrm{zu}$ beantworten. ${ }^{117}$ Da vorliegend keine entsprechende explizite Bestimmung vorliegt, ist die beanspruchte Regelungsweite im Wege der Auslegung zu ermitteln. Dabei ist im Sinne einer Schonung der Kompetenzen der Länder grundsätzlich nicht vom abschließenden Charakter auszugehen. ${ }^{118}$

117 Böhm DÖV 1998, 234 (235); Jarass NVwZ 1996, 1041 (1044); Oeter in: v. Mangoldt/Klein/ Starck, GG, Kommentar, 7. Aufl. 2018, Art. 72 Rn. 70 m.w. N.

118 Vgl. Böhm DÖV 1998, 234 (235); Jarass NVwZ 1996, 1041 (1043) und die weiteren Nachweise auch zur Rechtsprechung des BVerfG bereits oben in den Fn. $110 \mathrm{ff}$. 


\section{b) Klare systematische, teleologische und genetische Gründe für abschließende Regelung}

Die systematische, teleologische und auch genetische Auslegung sprechen vorliegend jedoch allesamt für einen abschließenden Charakter hinsichtlich der Frage der zulässigen Miethöhe. ${ }^{119}$ So werden auf Bundesebene sowohl für Bestands- als auch für Neuvermietungen die oben skizzierten (dazu III.1.), ausdifferenzierten Regelungen getroffen, die gerade die Miethöhe betreffen und die Interessen von Vermieter und Mieter zum Ausgleich bringen sollen. In beiden Fällen werden die Konkretisierungs- und Abweichungsmöglichkeiten der Länder zudem ganz genau benannt. Für Bestandsmieten wird nur eine Verschärfung der Kappungsgrenze der Erhöhungsmöglichkeiten der Mieten von 20\% auf 15\% innerhalb von drei Jahren eröffnet. Dafür müssen die Länder allerdings im Rahmen einer Rechtsverordnung gemäß §558 Abs. 3 S. 2 BGB zunächst feststellen, dass „die ausreichende Versorgung der Bevölkerung mit Mietwohnungen zu angemessenen Bedingungen in einer Gemeinde oder einem Teil einer Gemeinde besonders gefährdet ist“, und die jeweiligen Gebiete identifizieren. Noch stärker ist die Engführung bei Neuvermietungen angelegt: Hier müssen die Länder ebenfalls im Wege einer Rechtsverordnung entsprechende „Gebiete mit einem angespannten Wohnungsmarkt“ identifizieren. Eingrenzend ist aber darüber hinaus eine Absenkung des zulässigen „Aufschlags“ auf die Vergleichsmiete in Höhe von $10 \%$ nicht vorgesehen. Aus der systematischen Interpretation im Vergleich zur Kappungsgrenze für Bestandsmieten folgt daraus, dass hier keine Änderung hinsichtlich der Fixierung des Aufschlags durch die Länder gewünscht ist. Andernfalls hätte der Bundesgesetzgeber vorsehen müssen, dass der Aufschlag auch abgesenkt werden kann.

Damit wird der Regelungsansatz des Bundesgesetzgebers deutlich, der im sozial und wirtschaftlich empfindlichen Bereich der Mietenregulierung ein austariertes und abschließendes Konzept zur zulässigen Preishöhe entwickeln will. Könnten die Länder etwa die Kappungsgrenze bei Bestandsmieten oder den Aufschlag auf die Vergleichsmiete bei Neuvermietungen weiter absenken, ergäbe eine explizite Regelung der Option einer Absenkung der Kappungsgrenze bloß auf 15\% keinen Sinn.

Weitere Verschärfungen der bestehenden Regelung sind vom Bundesgesetzgeber explizit abgelehnt worden (siehe oben III.1.b) bb)). Damit wird der Wille des Bundes deutlich, am eingeschlagenen Weg festzuhalten und zugleich eine si-

119 So inzwischen auch BayVerfGH, Entsch. v. 16.7.2020 - Vf. 32-IX-20, BeckRS 2020, 16071, Rn. $58 \mathrm{ff}$. 
gnifikante Verschärfung ratione materiae im Übrigen abzulehnen. In der Gesetzesbegründung wird dies sogar explizit hervorgehoben. So heißt es unter Übernahme der Erkenntnisse einer vom DIW in Vorbereitung des Gesetzesentwurfs angefertigten Studie ${ }^{120}$, dass „stärker regulierende Eingriffe in die Preisbildung“ unterbleiben sollen, da sie sich negativ auf den Wohnungsmarkt auswirken könnten, und stattdessen „Änderungen an den Regelungen der ,Mietpreisbremse“ nur behutsam und mit der nötigen Vorsicht vorgenommen werden“ sollen. ${ }^{121}$ Zudem ist ein Antrag der Fraktion BÜNDNIS 90/DIE GRÜNEN, der weitere Verschärfungen ratione materiae in Richtung der Berliner Regelung (allerdings auch weit weniger radikal) vorgesehen hat, wie eine Absenkung des Zuschlags auf die ortsübliche Vergleichsmiete von 10\% auf 5\% und eine Streichung der Privilegierung für höhere Vormieten ${ }^{122}$, u.a. mit den Stimmen der Regierungsparteien abgelehnt worden ${ }^{123}$. Dieser ganze Streit wäre überflüssig, wenn die Länder, von deren Tätigwerden auch die Aktivierung der „Mietpreisbremse“ abhängt, ohnehin außerhalb des „Korsetts“ der BGB-Anforderungen per Gesetz regeln könnten, was sie wollten, also auch eine Absenkung auf einen Zuschlag in Höhe von 5\% im Fall der Neuvermietung.

\section{c) Gewollte Beschränkung der Gestaltungsmöglichkeiten der Länder}

Damit liegt eine abschließende Regelung des Bundes dahingehend vor, was die Länder als Eingriffe in die Mietpreishöhe im Rahmen der Nutzung der Verordnungskompetenzen in angespannten Wohnungsmärkten vornehmen dürfen, nämlich einerseits die Aktivierung der „Mietpreisbremse“ für Neuvermietungen nunmehr für weitere fünf und daher insgesamt zehn Jahre - sowie andererseits eine Absenkung der Kappungsgrenze von Mieterhöhungen von 20\% auf 15\%. Der Sinn dieser klar geregelten und damit ausdrücklichen Beschränkungen der Eingriffsmöglichkeiten der Länder wird in sein Gegenteil verkehrt, wenn daraus gerade abgeleitet wird, dass der Bundesgesetzgeber die zulässigen Miethöhen

120 DIW, Untersuchung der Wirksamkeit der in 2015 eingeführten Regelungen zur Dämpfung des Mietanstiegs auf angespannten Wohnungsmärkten (Mietpreisbremse), erstellt im Auftrag des BMJV, 2018, S. 42f., abrufbar im WWW unter der URL https://www.bmjv.de/SharedDocs/Down loads/DE/Ministerium/ForschungUndWissenschaft/MPB_Gutachten_DIW.pdf;jsessionid= BC2B170425388F37 A28E982 A09 A170D9.2_cid289?_blob=publicationFile\&v=2 (zuletzt abgerufen am 1.7.2020).

121 BT-Drs. 19/15824, S. 11.

122 BT-Drs. 19/15122.

123 Beschlussempfehlung BT-Drs. 19/17156. 
nicht abschließend regeln wollte. ${ }^{124}$ Diese Argumentation geht offensichtlich davon aus, dass es entweder nur eine abschließende Regelung ohne Gestaltungsmöglichkeiten der Länder oder eine nicht abschließende Regelung mit Gestaltungsmöglichkeiten der Länder gibt. Gäbe es nur diese zwei Möglichkeiten, wäre tatsächlich die zweite die Vorliegende. Warum es aber nicht drittens eine abschließende Regelung gerade der Gestaltungsmöglichkeiten der Länder geben sollte, leuchtet nicht ein. Genau diese Variante wurde vorliegend gewählt und nur diese Variante wird auch dem sinnvollen Regelungsanliegen des Bundesgesetzgebers gerecht, dass nämlich das komplexe Verfahren der Mietpreisfestsetzung über das Vergleichsmietenkonzept und die damit einhergehende Abwägung der Interessen der Mieter und Vermieter nur einmal konsistent vorgenommen werden kann. Eine von der Bundesregelung abweichende Austarierung auf Länderebene ist eben nicht eine weitere zusätzliche Regelung wie eine Nachbarschaftsregelung neben einer baurechtlichen Bestimmung ${ }^{125}$, sondern eine konterkarierende Regelung, die die Bundesregelung entwertet (dazu noch weiter bei 4.).

Ergänzend sei darauf hingewiesen, dass das BVerfG ${ }^{126}$ und auch die überwiegende Literatur ${ }^{127}$ davon ausgehen, dass die Landesverordnungsgeber nach $\S 556 \mathrm{~d}$ Abs. 2 BGB sogar tätig werden müssen, wenn Gebiete mit angespannten Wohnungsmärkten im Sinne der Vorschrift vorliegen. D.h. der Bund hat sein Regelungskonzept sogar soweit ausdifferenziert, dass das vorgesehene Schutzinstrumentarium bei Neuvermietungen greift, wenn ein angespannter Wohnungsmarkt vorliegt. Dieses Verständnis der landesrechtlichen Handlungspflicht betont besonders deutlich das umfassende und abschließende Handlungskonzept des Bundes.

124 Für eine vom Bundesgesetzgeber gewollte Beschränkung der Landesgesetzgeber außerhalb der eng umgrenzten Verordnungsvorbehalte inzwischen auch BayVerfGH, Entsch. v. 16.7.2020 Vf. 32-IX-20, BeckRS 2020, 16071, Rn. $64 \mathrm{ff}$.

125 So das Beispiel bei Kingreen Zur Gesetzgebungskompetenz der Länder für das Öffentliche Mietpreisrecht bei Wohnraum, 2020, Kurzgutachten für die Bundestagsfraktion DIE LINKE, S. 23, abrufbar im WWW unter der URL https://www.linksfraktion.de/fileadmin/user_upload/PDF_Do kumente/2020/200218_Kingreen_Mietendeckel_Kompetenz.pdf (zuletzt abgerufen am 1.7.2020). 126 BVerfG, Beschl. v. 18.7.2019-1 BvL 1/18, Rn. 111, abrufbar im WWW unter der URL http:// www.bverfg.de/e/lk20190718_1bvl000118.html - ECLI:DE:BVerfG:2019:1k20190718.1bvl000118 (zuletzt abgerufen am 1.7.2020).

127 Lange DVBl. 2015, 1551 (1557); Lehmann-Richter WuM 2015, 204 (205); Schuldt Mietpreisbremse. Eine juristische und ökonomische Untersuchung der Preisregulierung für preisfreien Wohnraum, 2017, S. 244ff.; a. A. Hinz in: Dauner-Lieb/Langen, BGB-Schuldrecht, 3. Aufl. 2016, $\S 556 d$ BGB Rn. 13. 


\section{d) Keine abweichenden Erkenntnisse aus der Rechtsprechung des Bundesverfassungsgerichts (insbesondere im Beschluss vom 23. März 1965)}

Auch der Beschluss des Zweiten Senats des BVerfG vom 23. März 1965 bestätigt im Übrigen keineswegs die angedeutete, schon sachlogisch, aber auch teleologisch wenig vertretbare Beschränkung des Bundesgesetzgebers im Rahmen der Ausübung einer konkurrierenden Gesetzgebungskompetenz auf die Alternativen, dem Landesgesetzgeber entweder überhaupt keine landesrechtlichen Konkretisierungsspielräume einzuräumen oder ihn durch die Einräumung von beschränkten Verordnungsermächtigungen zugleich zu einem Handeln auch jenseits der Beschränkungen und dabei zum Erlass auch gegen den Regelungssinn dieser Beschränkungen und der übrigen Bundesregelungen zu ermächtigen. ${ }^{128}$ Denn in dem zugrunde liegenden Fall aus dem Jahr 1960 ging es um das Ausfüllen von Strafrechtsnormen des Bundes durch die dazu in der Bundesregelung explizit befugte Landesregierung und sodann um die normkategoriale Frage, ob das anschließende Nutzen einer entsprechenden Verordnungsermächtigung durch die Landesregierung eine Landes- oder eine Bundesnorm generiert. Diese, 60 Jahre später seltsam trivial anmutende Frage hat das BVerfG seinerzeit zu Recht im Sinne des Vorliegens einer Landesnorm bejaht. ${ }^{129}$ Vorliegend ist es auch völlig unstreitig, dass das Ausfüllen der Verordnungsermächtigungen im BGB auf der Landesebene zu Landesnormen führt. Vielmehr stellt sich hier ja die Frage, ob die Länder über eine Gesetzgebungskompetenz für eine Regelung jenseits der im Bundesrecht eröffneten Befugnisse in den Verordnungsermächtigungen verfügen - und zwar durch die Schaffung von Landesgesetzen im formellen Sinne. Dazu lässt sich der Entscheidung des BVerfG nichts entnehmen, jedenfalls nicht die oben aufgezeigte unsinnige Beschränkung des Handlungsspielraums des Bundes mit Blick auf die Eröffnung von Gestaltungsmöglichkeiten der Länder auf ein „Ganz oder gar nicht“.

Die zitierte Passage des BVerfG u.a. dahin gehend, dass der „Bundesgesetzgeber die Materie nicht erschöpfend geregelt hat, weil er sie im Hinblick auf die Verschiedenheit der örtlichen Verhältnisse nicht erschöpfend regeln konnte“ ${ }^{130}$,

128 So aber Kingreen Zur Gesetzgebungskompetenz der Länder für das Öffentliche Mietpreisrecht bei Wohnraum, 2020, Kurzgutachten für die Bundestagsfraktion DIE LINKE., S. 15 f., abrufbar im WWW unter der URL https://www.linksfraktion.de/fileadmin/user_upload/PDF_Doku mente/2020/200218_Kingreen_Mietendeckel_Kompetenz.pdf (zuletzt abgerufen am 1.7.2020) unter Hinweis auf BVerfGE 18, 407 (417).

129 BVerfGE 18, 407.

130 BVerfGE 18, 407, Rn. 36 - juris. 
gibt zutreffend die strafrechtliche Regelungssituation wieder. Vorliegend wird auch niemand behaupten wollen, der Bund habe den Rechtsrahmen zur Regelung der Höhe der Mietpreise in dem Sinne abschließend regeln wollen, dass die Länder gar nichts mehr tun können. Genau das Gegenteil ist der Fall: Der Bund hat den Bundesländern ja gerade eine entsprechende Konkretisierungsbefugnis eingeräumt und wollte die Materie tatsächlich insoweit, aber eben auch nur insoweit (!), nicht abschließend regeln. Abschließend geregelt sind also die Spielräume der Länder, unter welchen Voraussetzungen sie welche Eingriffe in die Mietpreishöhen vornehmen können - Absenkung der Kappungsgrenze in Bestands- und „Mietpreisbremse“ in Neuvermietungsverhältnissen. Unter Wahrung der Treue im Bund-Länder-Verhältnis möchte der Bundesgesetzgeber den Ländern nicht weniger, aber eben auch nicht mehr zugestehen (dazu auch sogleich 4. und insbesondere 4.d)). Die diesbezügliche Einschränkungskompetenz des Bundes wird im Übrigen auch vom BVerfG gerade in der angeführten Entscheidung aus den 1960er Jahren betont. ${ }^{131}$ Daher wird in der Literatur, sofern sie sich damit näher auseinandersetzt, auch davon ausgegangen, dass die Einräumung einer Ermächtigungsnorm zum Erlass von Verordnungen zugunsten eines Landesverordnungsgebers für die Länder, insbesondere auch die Landesgesetzgeber, eine Bindung an die Vorgaben und den Rahmen der Verordnungsermächtigung zur Folge hat. ${ }^{132}$ Eine im Übrigen und insoweit abschließende Regelung ist also durch die Verordnungsermächtigung zugunsten der Landesregierungen gerade nicht ausgeschlossen, sondern wird durch ihre Konditionierung vielmehr belegt.

\section{Gesetzgebungskompetenz der Länder zur Mietpreisregelung nach Art. 74 Nr. 18 GG?}

Durch die Föderalismusreform I aus dem Jahr 2006 ist die konkurrierende Gesetzgebungskompetenz für den Titel „Wohnungswesen“ im vormaligen Art. 74 Nr. 18 GG gestrichen worden. Daraus wird zum Teil sehr weitgehend abgeleitet, dass das Mietpreisrecht damit umfassend in die Gesetzgebungskompetenz der Länder nach Art. 70 GG gefallen sei, ${ }^{133}$ während teilweise umgekehrt ebenfalls

131 Ebenda.

132 Oeter in: v. Mangoldt/Klein/Starck, GG, Kommentar, 7. Aufl. 2018, Art. 72 Rn. 82; Uhle in: Maunz/Dürig, Grundgesetz-Kommentar, Art. 72 Rn. 101, 76. Ergänzungslieferung (Stand: Dezember 2015); Wollenschläger in: Bonner Kommentar, GG, Art. 72 Rn. 209, 192. Ergänzungslieferung (Stand: August 2018).

133 So für ein „öffentliches Mietpreisrecht“ Weber JZ 2018, 1022 (1027); ders. ZMR 2019, 389 (392ff.); dazu ausführlich Kingreen Zur Gesetzgebungskompetenz der Länder für das Öffentliche 
sehr weitgehend davon ausgegangen wird, dass eine abschließende Kompetenz des Bundes zur Regelung des Mietpreisrechts im bürgerlichen Recht getroffen worden sei. ${ }^{134}$

Für die fehlende Übernahme der Kompetenz des Mietpreisrechts als Bestandteil des Wohnungswesens spricht, dass in der - abschließend formulierten Aufzählung der „Bereiche des Wohnungswesens“ in der Gesetzesbegründung zur Verfassungsänderung vieles, aber nicht mehr das Mietpreisrecht auftaucht. ${ }^{135}$ Allerdings gehörte das öffentliche Mietpreisrecht jedenfalls zum Instrumentarium der „Wohnungszwangswirtschaft“ unter dem bisherigen Kompetenztitel des „Wohnungswesens“136, was im Rahmen einer historischen und an der Gesetzgebungspraxis orientierten Interpretation für die Annahme einer unter Art. 70 GG fortbestehenden Kompetenz der Länder spricht. ${ }^{137}$ Umgekehrt kann der Umstand, dass zur Zeit der Föderalismusreform 2006 das öffentliche Mietpreisrecht durch das soziale Mietrecht im BGB abgelöst worden war und auch stark in der Tradition der Wohnungsnot der Nachkriegszeit mit entsprechend notwendigen drastischen Zwangsmaßnahmen der Wohnraumbewirtschaftung aufgrund des flächigen (!) Wohnungsmangels in Deutschland stand, dafür sprechen, dass insoweit auf der Landesebene keine Regelungsnotwendigkeit mehr gesehen wurde. Es ist auch nicht ersichtlich, dass es noch entsprechende Regelungen gab, die unter der Kompetenzverteilung nach der Föderalismusreform 2006 hätten fortgeschrieben werden müssen. ${ }^{138}$ Vor diesem Hintergrund erklärt sich auch die mangelnde

Mietpreisrecht bei Wohnraum, 2020, Kurzgutachten für die Bundestagsfraktion DIE LINKE., S. 9 ff., abrufbar im WWW unter der URL https://www.linksfraktion.de/fileadmin/user_upload/ PDF_Dokumente/2020/200218_Kingreen_Mietendeckel_Kompetenz.pdf (zuletzt abgerufen am 1.7.2020).

134 So Papier Landeskompetenz zur Einführung eines sogenannten Mietendeckels? Rechtsgutachtliche Stellungnahme im Auftrag des Bundesverbandes Deutscher Wohnungs- und Immobilienunternehmen e.V. - GdV, September 2019, S. 6-8 und vor allem S. 13 und 14, abrufbar im WWW unter der URL https://web.gdw.de/uploads/pdf/Pressemeldungen/Gutachten_Mietende ckel_Zustaendigkeit.pdf (zuletzt abgerufen am 1.7.2020).

135 BT-Drs. 16/813, S. 13.

136 Siehe insbesondere den Entwurf eines Gesetzes über den Abbau der Wohnungszwangswirtschaft und über ein soziales Mietrecht vom 6.8.1959, BT-Drs. 03/1234, S. 46.

137 So insbesondere Kingreen Zur Gesetzgebungskompetenz der Länder für das Öffentliche Mietpreisrecht bei Wohnraum, 2020, Kurzgutachten für die Bundestagsfraktion DIE LINKE., S. 9 ff., abrufbar im WWW unter der URL https://www.linksfraktion.de/fileadmin/user_upload/ PDF_Dokumente/2020/200218_Kingreen_Mietendeckel_Kompetenz.pdf (zuletzt abgerufen am 1.7.2020).

138 Daher ist auch das von Kingreen aaO, S. 11, angeführte systematische Argument nicht zwingend, dass keine explizite Lösung im Grundgesetz für den Fortbestand öffentlich-rechtlicher Mietpreisregelungen der Länder geschaffen worden sei, da derartige Regelungen im Jahr 2006 
Diskussion, da im Jahr 2006 niemand vorhersehen konnte, dass eines Tages wieder Instrumente diskutiert werden, die eher in Krisenzeiten wie der Nachkriegszeit angemessen erschienen. ${ }^{139}$

Daher sprechen sehr gute Gründe dafür, dass die Mietpreisregulierung im Zuge der Föderalismusreform im sozialen Mietrecht des BGB aufgegangen ist und die Länder im Bereich des Wohnungswesens noch viele Gestaltungsmöglichkeiten haben, aber eben nicht das Instrument einer Miethöhenregulierung. Die Gesetzesbegründung zur Föderalismusreform verweist auf die Fülle der verbleibenden Gestaltungsmöglichkeiten, die von den Ländern auch umfassend genutzt werden, vom wichtigen Recht der sozialen Wohnraumförderung zum steuerungspräziseren Schutz einkommensschwacher Haushalte bis hin zum angebotswahrenden Zweckentfremdungsrecht im Wohnungswesen.

Aber selbst wenn die Länder eine entsprechende Kompetenz auch nach der Föderalismusreform behalten hätten, enthält das BGB jedenfalls unstreitig Regelungen des Mietpreisrechts, die nach der Föderalismusreform vom Bundesgesetzgeber auch noch im Rahmen der „Mietpreisbremse“ ergänzt wurden, ohne dass das BVerfG insoweit Kompetenzprobleme mit Blick auf die gesetzlichen Regelungen erkannt hätte. Eine konkurrierende Gesetzgebungskompetenz des Bundes liegt also unstreitig für Mietpreisregelungen vor (dazu bereits oben 1.). Entscheidend ist damit vorliegend, eine Kompetenz der Länder für das Wohnungswesen unterstellt, ob der Bund abschließende Regelungen zur Mietpreishöhe kompetenzkonform treffen durfte und dies auch wollte (was der Fall ist, siehe bereits oben 2.), und ob die Berliner Landesregelung diese missachtet (was ebenfalls der Fall ist, dazu sogleich 4.).

\section{Verschärfende und konterkarierende Wirkung der Berliner Regelungen}

Die Berliner Regelungen betreffen von den Regelungszielen und -wirkungen identische Regelungsgegenstände. Das gilt in Bezug auf die Regelungen des Landes Berlin sowohl hinsichtlich des Mietenstopps (dazu a)) und der Absenkungsmöglichkeit der Bestandsmieten als auch hinsichtlich der Mietobergrenzen für Neuvermietungen (dazu b)). Den Berliner Regelungen liegt auch kein grund-

nicht ersichtlich sind. Im Übrigen wäre die Konsequenz die, dass entsprechende Regelungen aufzuheben wären.

139 Anders insoweit Kingreen aaO, S. 10, der aus dem diesbezüglichen Schweigen ein Argument dafür ableitet, dass keine Änderungen gegenüber dem bisherigen (historisch aber überholten) Verständnis gewollt sein konnten. 
sätzlich abweichendes Regelungskonzept „,auf dem Gebiet des Wohnungswesens“ zugrunde (dazu c)), so dass auch keine Rücksichtnahme auf die Bundesregelung erkennbar ist, ebenso wenig wie eine widerspruchsfreie Ergänzung der Bestimmungen auf Bundesebene vorliegt, sondern deren Aushöhlung und Konterkarierung (dazu d)).

\section{a) Verschärfte Regelungswirkung des Mietenstopps nach § 3 MietenWoG Bln im Vergleich zur Kappungsgrenze im BGB}

Der Mietenstopp nach $\S 3$ Abs. 1 MietenWoG Bln sieht hinsichtlich der Bestandsmieten vor, dass jede Miete, die die zum Stichtag des 18. Juni 2019 wirksam vereinbarte Miete überschreitet, grundsätzlich verboten ist. Er löst damit eine in der Grundstruktur identische Wirkung aus wie die Kappungsgrenze des §558 Abs. 3 BGB in Höhe von $20 \%$ bzw. $15 \%$, nur dass er ungleich radikaler ist. ${ }^{140}$ Hätte der Bund in $\S 558$ Abs. 3 BGB eine Öffnungsklausel vorgesehen, dass die Länder die Kappung nicht nur auf $15 \%$ in drei Jahren, sondern auch auf $0 \%$ für eine begrenzte Zahl von maximal zwei Jahren und eine anschließende Inflationsanpassung, jedoch allerhöchstens um 1,3\% pro Jahr, absenken können, wäre § 3 MietenWoG Bln als Landesregelung von der Gestaltungsermächtigung des Bundesgesetzes gedeckt. Auch wäre es denkbar gewesen, dass der Bundesgesetzgeber verschiedene Konzepte einer Absenkung der Kappungsgrenze eröffnet hätte. All dies ist aber gerade nicht der Fall. Der Bund wollte den Korridor einer ausgewogenen Mietpreishöhenregulierung also selbst bestimmen und den Ländern nur einen sehr begrenzten Spielraum eröffnen, die Kappungen zu verschärfen. Dieser wird ganz offensichtlich gesprengt und letztlich überflüssig gemacht, wenn der Landesgesetzgeber Berlins nicht nur - wie bereits zulässigerweise erfolgt - die Kappungsgrenze im Rahmen einer Verordnung auf 15\% in drei Jahren absenkt, sondern auf $0 \%$ zuzüglich eines äußerst beschränkten Inflationsausgleichs.

\section{b) Verschärfende Wirkung des $\S 4$ MietenWoG Bln (Mietobergrenzen) und $\S 5$ MietenWoG Bln (Überhöhte Mieten)}

Nach $\S 4$ MietenWoG Bln werden Mietobergrenzen für Neuvermietungen anhand einer Mietentabelle ( $§ 6$ MietenWoG Bln) eingeführt und höhere Mieten verboten. Die Berliner Regelung erstreckt diese Wirkung durch das Verbot höherer Mieten

140 So auch Wolfers/Opper DVBl. 2019, 1446 (1448). 
als solcher, die in der Mietentabelle nach $\S 6$ (bzw. ergänzend nach $\S 7$ ) MietenWoG Bln vorgesehen sind, in $\S 5$ MietenWoG Bln in Verschärfung der Vorgaben im BGB auch auf Bestandsmietverhältnisse. In Abweichung zur Regelung im BGB wird als Vergleichsmaßstab im Übrigen wiederum nicht auf ein dynamisches Vergleichsmietenmodell rekurriert, sondern auf ein statisches Miettabellen-Konzept. Die Miettabelle wurde zwar im Ausgangspunkt aus dem Berliner Mietenspiegel von 2013 entwickelt, in entscheidender Abweichung vom Berliner Mietenspiegel wird aber die Lage der Wohnung nicht vollumfänglich und auch nur für den Fall der Mietenabsenkung berücksichtigt. In der Folge sind die mehr als 20\% höheren Bestandsmieten abzusenken, da diese zivilrechtlich verboten (§ 134 BGB) und im Übrigen mit einem Ordnungswidrigkeitstatbestand sanktionsbewehrt worden sind (§ 11 Abs. 1 Nr. 4 MietenWoG Bln).

Damit unterscheidet sich die Regelung von der Regulierung der Bestandsmieten im BGB in ihrer Eingriffswirkung deutlich. So liegt eine erhebliche Verschärfung darin begründet, dass nicht mehr auf eine dynamische Vergleichsmiete (nach Mietenspiegel) abgestellt wird, sondern auf fixe Werte einer Mietentabelle, die sich lediglich am Berliner Mietenspiegel 2013 orientieren. Deutlich verschärfend wirkt ferner, dass die Regelung auch die Notwendigkeit der Anpassung im Sinne einer Absenkung - bereits konsentierter Mieten vorsieht. So war das gesamte Mietpreisregulierungsrecht des BGB bislang darauf angelegt, dass im Rahmen einer einvernehmlichen Vereinbarung nach $\S 557$ Abs. 1 BGB jederzeit ein erhöhter Mietzins vollkommen unabhängig von der Höhe etwaiger Vergleichsmieten vereinbart werden kann. Das ist schon vor dem Hintergrund wichtig, dass es angesichts regelmäßig fehlender qualifizierter Mietspiegel und auch unabhängig davon oftmals begründeten Anlass zum Streit darüber gibt, von welcher Vergleichsmiete auszugehen ist. Von diesem bisherigen Grundsatz ist der Bundesgesetzgeber allerdings schon für Neuvermietungen im Rahmen der „Mietpreisbremse“ insoweit abgewichen, als er bestimmt hat, dass nach $\S 556 \mathrm{~g}$ Abs. 1 BGB nunmehr auch Mieten, die oberhalb der zulässigen 10\%igen Abweichung von der Vergleichsmiete liegen, unwirksam sind. Überhöhte Mietzahlungen können sodann im Wege des Bereicherungsrechts zurückverlangt werden. Damit wird in Neuvermietungsverhältnissen eine Mietenabsenkung auf die zulässige Höhe bewirkt. Dasselbe bewirkt die Mietobergrenzen-Regulierung nach $\S 4$ MietenWoG Bln und verschärft die Wirkung, da eine (niedrigere) gesetzlich fixierte Miete nach Mietentabelle Referenzmaßstab ist und kein $10 \%$ iger Zuschlag greift.

Dadurch und aufgrund des statischen Ansatzes verschärft sich die Eingriffswirkung der Berliner Regelung massiv ebenso wie der Entkoppelungseffekt von der Marktmiete besonders gravierend ist. Gleichwohl ist die Wirkungsrichtung und die Funktionsweise der Berliner Regelung mit der im BGB vergleich- 
bar. ${ }^{141}$ Auch hier gilt aber wiederum, dass der Bund weder bei den Neu- noch bei den Bestandsmieten eine Öffnungsklausel für einen derartig radikalen Eingriff in die Preisbildung vorgesehen hat. Insoweit verschärft das in der Berliner Regelung vorgesehene Instrument der Mietenabsenkung die Bestimmungen im BGB, macht sie wiederum weitgehend überflüssig und konterkariert das in der BGB-Regelung beabsichtigte Ziel eines angemessenen Ausgleichs von Mieter- und Vermieterinteressen. Eine entsprechende Parallele lässt sich insbesondere zwischen der Mietobergrenze für Neuvermietungen nach §4 MietenWoG Bln und der ,Mietpreisbremsen“-Regelung der $§ \S 556 d$ - g BGB ziehen. Ähnlich dem Mietenstopp für Bestandsmieten ist die Berliner Regelung in der Funktionsweise identisch, gleichzeitig aber quantitativ deutlich radikaler, da der Vergleichsmaßstab aufgrund der statischen Ausrichtung auf die Mietentabelle abgesenkt wird und zudem ein „Sicherheits“-Zuschlag entfällt. Damit liegt wiederum eine deutliche Verschärfung vor, für die im Bundesgesetz aber keine Öffnungsmöglichkeit angelegt ist. §4 MietenWoG Bln verschärft die noch einigermaßen ausbalancierte Bundesregelung radikal zu Lasten der Vermieter, macht die Bundesregelung wiederum überflüssig und konterkariert deren Konzept.

\section{c) Kein abweichendes Regelungskonzept „auf dem Gebiet des Wohnungswesens“}

Die Berliner Regelung enthält dabei kein in einem verfassungsrechtlichen Sinne kompetenzbegründendes abweichendes Regelungskonzept „auf dem Gebiet des Wohnungswesens“, sondern verschärft die Ansätze im BGB mit einer identischen Stoßrichtung. Der konzeptionelle Unterschied besteht daher lediglich in der ungewöhnlichen Radikalität, aber nicht in einem wie auch immer gearteten eigenständigen Regelungskonzept für einen abgetrennten Lebenssachverhalt in Form etwa eines abtrennbaren Marktes. Insoweit genügen keine denkbaren Verbindungslinien $\mathrm{zu}$ einem noch $\mathrm{zu}$ entwickelnden Wohnraumbewirtschaftungskonzept (dazu aa)). Es hilft auch nicht, dass die Berliner Regelung gegebenenfalls stärker öffentlich-rechtlich geprägt ist als jene im BGB (dazu bb)). Schließlich ergibt sich nichts Anderes vor dem Hintergrund sonstiger verfassungsrechtlicher Vorgaben, etwa in der Berliner Landesverfassung (dazu cc)).

141 So auch Wolfers/Opper DVBl. 2019, 1446 (1448): „Zwischen Mietpreisbremse und Mietpreisdeckel besteht daher kein qualitativer, sondern lediglich ein quantitativer Unterschied. Der in Berlin geplante Mietendeckel ist sachlich eine Mietpreisbremse, nur eine besonders starke und besonders starre.“ 


\section{aa) Wohnungspolitische Ziele und Verbindungslinien in entsprechenden Konzepten nicht kompetenzbegründend}

Teilweise wird eine Landeskompetenz zu der ganzen Breite oder einzelnen mietpreisregulierenden Vorschriften damit begründet, dass sie Bestandteil eines „wohnungspolitischen Konzepts“ seien, um einen ausgeglichenen Wohnungsmarkt wiederherzustellen. ${ }^{142}$ Das gelte zumindest für das mit dem Mietenstopp erfolgende zeitlich befristete „Mietenmoratorium““ ${ }^{433}$ Eine derartige Argumentation verkennt jedoch die identische Regelungsrichtung von Mietpreisregelungen im BGB und „Mietendeckel“ einschließlich „Mietenmoratorium“ im MietenWoG Bln. Die Instrumente unterscheiden sich lediglich in ihrer Radikalität der Abkopplung vom freien und unregulierten Mietenmarkt, nämlich noch vergleichsweise moderat im Fall der BGB-Regelung, hingegen radikal im Fall der Berliner Regelung (dazu bereits oben a) und b)). Wenn für ein Regelungsinstrument aber eine konkurrierende Bundesgesetzgebungskompetenz besteht und der Bund davon Gebrauch macht, darf ein Land nicht konterkarierende Regelungen treffen (dazu ebenfalls bereits oben 1. und 2.) mit dem Argument, dieses Instrument könne zugleich im Rahmen eines weiteren Gesamtinstrumentariums auf Landesebene zum Einsatz kommen. Insofern hinken auch die historischen Vergleiche, dass es auf Landesebene bereits Mietenmoratorien gegeben habe. ${ }^{144}$ Dies ist kompetenzrechtlich auch bislang angesichts der konkurrierenden Gesetzgebungskompetenz möglich gewesen. Voraussetzung war aber vor wie nach der Föderalismusreform, dass diese nicht einer Bundesregelung widersprachen. Es ist auch nicht ersichtlich, dass das seinerzeit der Fall gewesen wäre.

Entscheidend ist damit, wie auch der Wissenschaftliche Dienst des Bundestages in seiner gutachterlichen Stellungnahme zutreffend ausführt ${ }^{145}$, ob ein Wohnraumbewirtschaftungskonzept (als scharfe Eingriffsvariante zur Beseitigung der eklatanten Wohnungsnot nach der Katastrophe und den Ausbombungen des Zweiten Weltkriegs) bzw. (als weit weniger eingriffsintensive Variante) ein öffentlich geförderter Wohnraum vorliegt. In diesen beiden Fällen sind die Miet-

142 Battis Verfassungsrechtliche Prüfung des Referentenentwurfs eines Gesetzes zur Neuregelung gesetzlicher Vorschriften zur Mietenbegrenzung in Berlin (Berliner MietenWoG), Rechtsgutachten im Auftrag der Senatskanzlei des Landes Berlin, S. 17, abrufbar im WWW unter der URL https://www.berlin.de/rbmskzl/aktuelles/fuer-alle/191004-rechtsgutachten-mietendeckel.pdf (zuletzt abgerufen am 1.7.2020).

143 So differenzierend insbesondere Battis aaO, S. $14 \mathrm{ff}$.

144 Dazu etwa Battis aaO, S. 11 und passim.

145 Wissenschaftlicher Dienst des Deutschen Bundestages, Gesetzliche Mietpreisregulierung durch die Länder aufgrund der Gesetzgebungskompetenz für das Wohnungswesen, WD 33000 -029/19 vom 5.2.2019, S. 3 ff. 
objekte - in unterschiedlichem Umfang - dem freien Mietmarkt entzogen und können auch einer verschärften Mietpreisregulierung unterworfen werden, ohne dass es zu einem Widerspruch mit der Mietenregulierung des Bundesgesetzgebers kommt. In Bezug auf „frei am Wohnungsmarkt angebotene Mietwohnungen“ gilt hingegen das zutreffende Verdikt des Wissenschaftlichen Dienstes des Bundestages, dass „das Mietpreisbindungsgesetz des Bundes eine abschließende gesetzliche Regelung“ geschaffen hat. ${ }^{146}$

Den Ländern sind damit auch nicht die Spielräume zur Einführung eines wohnungspolitischen Konzepts genommen, da sie in einer Übergangsphase etwa durch Mietzuschüsse für einkommensschwache Haushalte ganz gezielt die angestrebten Zwecke verfolgen können. Derweil bleibt es ihnen im Rahmen der übrigen verfassungsrechtlichen Grenzen kompetenzrechtlich unbenommen, ein umfassendes wohnungspolitisches Konzept $\mathrm{zu}$ entwickeln, das die gewünschten politischen Ziele über den Aufkauf von Belegungsrechten oder Wohnungen, die landeseigenen Wohnungsgesellschaften oder stärker intervenierende Wohnraumbewirtschaftungskonzepte verfolgt.

\section{bb) Öffentlich-rechtliche Teilüberformung nicht kompetenzbegründend}

Auf dieser Linie ist auch die teilweise vertretene Ansicht, die Kompetenz richte sich danach, ob die Mietpreisregulierung öffentlich-rechtlich (dann Zuständigkeit der Länder) oder zivilrechtlich (dann Zuständigkeit des Bundes) ausgestaltet sei, ${ }^{147}$ zurückzuweisen. So ist hinsichtlich des öffentlich-rechtlichen Charakters der Berliner Regelung zunächst darauf hinzuweisen, dass die Wirkung der Regelungen erst recht in der schlussendlich Gesetz gewordenen Fassung primär zivilrechtlich hergestellt wird: So sind dem Mietenstopp widersprechende Mieten nach $\S 3$ Abs. 1 MietenWoG Bln und $\S 5$ Abs. 1 MietenWoG Bln verboten bzw. unzulässig. Damit wird die zivilrechtliche Vertragsfreiheit regulatorisch in derselben Weise überformt wie bei der Kappungsgrenze und der „Mietpreisbremse“ nach

146 Wissenschaftlicher Dienst des Deutschen Bundestages, aaO, S. 6.

147 Besonders prononciert Mayer/Artz Öffentlich-rechtliche und privatrechtliche Aspekte eines Mietendeckels für das Land Berlin, Rechtsgutachten für die Fraktion der SPD im Abgeordnetenhaus von Berlin, 16.3.2019, S.19ff., abrufbar im WWW unter der URL https://www.spdfraktion-ber lin.de/system/files/mayer_artz_gutachten_mietendeckel_fuer_spd-fraktion.pdf; ähnlich FischerLescano/Gutmann/Schmid Landeskompetenzen für Maßnahmen der Mietpreisregulierung, Rechtsgutachten für die Rosa Luxemburg Stiftung, 2019, S. 10 ff., abrufbar im WWW unter der URL https://www.rosalux.de/fileadmin/rls_uploads/pdfs/Studien/Studien_8-19_Mietpreisregulie rung_web.pdf (zuletzt abgerufen jeweils am 1.7.2020); Putzer NVwZ 2019, 283 (284); Weber JZ 2018, 1022 (1022ff.). 
dem BGB: Im Fall der Berliner Regelung verstößt eine unangemessene Miethöhe gegen ein außerhalb des BGB normiertes Verbotsgesetz, das erst über die BGBVorschrift des $§ 134$ eine Wirkung im Vertragsverhältnis erlangt und grundsätzlich nicht durch ein „öffentlich-rechtliches“ Instrument durchgesetzt werden soll. Das ursprünglich vorgesehene öffentlich-rechtliche Absenkungsinstrumentarium bei Überschreitung der zulässigen Grenzen nach der Mietentabelle gemäß einer Vorgängerfassung des $\S 4$ i.V.m. den $\S \S 5$ und 6 MietenWoG Bln (Fassung vom 28. November 2019) ist durch den am 30. Januar 2020 beschlossenen Änderungsantrag vom 23. Januar 2020 gestrichen worden. ${ }^{148}$ Stattdessen ist nur noch eine Residualkompetenz der zuständigen Behörde in $\S 5$ Abs. 2 MietenWoG Bln vorgesehen (,Sie kann von Amts wegen alle Maßnahmen treffen, die insoweit zur Durchsetzung erforderlich sind“). Das bedeutet, dass grundsätzlich nur noch dann, wenn der Vermieter einen Härtefall geltend macht und das verwaltungsrechtliche Handlungsinstrumentarium der Härtefallgenehmigung nach § 8 MietenWoG Bln greift, eine öffentlich-rechtliche Regelung vorliegt. Selbst wenn es also auf den öffentlich-rechtlichen Charakter ankäme, wäre das Vorliegen einer hinreichenden öffentlich-rechtlichen Überformung im Kern zu verneinen.

Auf den öffentlich-rechtlichen Charakter kann es jedoch unter Kompetenzverteilungsgesichtspunkten keineswegs ankommen. Andernfalls hätte es der Landesgesetzgeber in der Hand, ein identisches Instrument des Bundes - die Regulierung der Höhe des Mietzinses - mit identischen Zwecken (Bereitstellung von Wohnraum zu angemessenen Preisen und Bekämpfung der Verdrängung „wirtschaftlich weniger leistungsfähiger Bevölkerungsgruppen aus stark nachgefragten Wohnquartieren“) faktisch funktionslos zu machen und zu konterkarieren, indem er es inhaltlich verschärft (u.a. in Form der Beschränkung der Erhöhungsmöglichkeiten von 15\% in drei Jahren auf 0\% plus verzögerten Inflationsausgleich) sowie (inzwischen sehr abgeschwächt) „öffentlich-rechtlich“ ergänzt und umformt durch die Option behördlicher Interventionen und der Verhängung von Bußgeldern. Die Stärkung des öffentlich-rechtlichen Charakters einer Regelung ist also keine hinreichende Bedingung für die Kompetenzkonformität einer Regelung durch die Länder. ${ }^{149}$

148 Ziff. 1f) des Änderungsantrags der dringlichen Beschlussempfehlung des Ausschusses für Stadtentwicklung und Wohnen vom 22.1.2020, AGH-Drs. 18/2437.

149 Ebenso im Ergebnis etwa Papier Landeskompetenz zur Einführung eines sogenannten Mietendeckels? Rechtsgutachtliche Stellungnahme im Auftrag des Bundesverbandes Deutscher Wohnungs- und Immobilienunternehmen e.V. - GdV, September 2019, insbesondere S. $10 \mathrm{ff}$,, abrufbar im WWW unter der URL https://web.gdw.de/uploads/pdf/Pressemeldungen/Gutachten_ Mietendeckel_Zustaendigkeit.pdf (zuletzt abgerufen am 1.7.2020); ferner u.a. Schede/Schuldt 


\section{cc) Kein anderes Ergebnis wegen Vorgaben der Berliner Verfassung}

Ferner sei noch darauf hingewiesen, dass auch aus der Berliner Verfassung und den dort angelegten Vorgaben zur Schaffung von Wohnraum keinerlei abweichende Ergebnisse folgen. ${ }^{150}$ So normiert Art. 28 Abs. 1 der Berliner Verfassung: „Jeder Mensch hat das Recht auf angemessenen Wohnraum. Das Land fördert die Schaffung und Erhaltung von angemessenem Wohnraum, insbesondere für Menschen mit geringem Einkommen, sowie die Bildung von Wohnungseigentum. "Schon an der Förderverpflichtung wird deutlich, in welche Richtung die Versorgungsverpflichtung des Landes geht, nämlich in die der Schaffung von Wohnraum, also in dessen Vermehrung. Dieses Ziel wird durch die ergriffenen Maßnahmen eher konterkariert. ${ }^{151}$ Ohne die Frage näher zu vertiefen, ob die Maßnahmen daher nicht eher gegen die Zielbestimmungen der Berliner Verfassung verstoßen, rechtfertigen diese Zielbestimmungen jedenfalls keine kompetenzwidrigen Maßnahmen. ${ }^{152}$ So verbleiben dem Land eine Fülle von kompetenzkonformen Handlungsmöglichkeiten bis hin zu Zuschüssen für einkommensschwache Haushalte. Letztlich steht die Kompetenzverteilung des Grundgesetzes zudem normenhierarchisch über der Berliner Verfassung.

\section{d) Keine Rücksichtnahme auf Bundesregelung}

\section{aa) Widersprüchlichkeit zu BGB-Vorschriften}

Nicht nachvollziehbar ist darüber hinaus das Argument, es läge „keine Widersprüchlichkeit“ vor, da ja „das mietrechtliche Schuldverhältnis unberührt“ bleibe, denn die Berliner Regelung „beschneidet lediglich die Möglichkeiten der

NVwZ 2019, 1572 (1574), mit dem zutreffenden Hinweis, dass die Berliner Regelungen ,in der Sache privatrechtsgestaltend wirken sollen“.

150 So aber offensichtlich Mayer/Artz Öffentlich-rechtliche und privatrechtliche Aspekte eines Mietendeckels für das Land Berlin, Rechtsgutachten für die Fraktion der SPD im Abgeordnetenhaus von Berlin, 16.3.2019, S. 30, abrufbar im WWW unter der URL https://www.spdfraktion-ber lin.de/system/files/mayer_artz_gutachten_mietendeckel_fuer_spd-fraktion.pdf (zuletzt abgerufen am 1.7.2020).

151 Siehe dazu oben III 1 und IV 2.

152 Ebenso etwa Papier Landeskompetenz zur Einführung eines sogenannten Mietendeckels? Rechtsgutachtliche Stellungnahme im Auftrag des Bundesverbandes Deutscher Wohnungs- und Immobilienunternehmen e.V. - GdV, September 2019, S. 14, abrufbar im WWW unter der URL https://web.gdw.de/uploads/pdf/Pressemeldungen/Gutachten_Mietendeckel_Zustaendigkeit. pdf (zuletzt abgerufen am 1.7.2020). 
Vermietenden zur Mieterhöhung. “153 Dies entspricht genau der Wirkungsweise der Regelung des $\S 558$ Abs. 3 BGB und der weiteren oben (dazu III.1.a)) geschilderten Regelungen des BGB. Schon das BGB sieht also Bestimmungen vor, die aus Gründen des sozialen Mieterschutzes, der Verhinderung von Gentrifizierung und der Wahrung des Zugangs von einkommensschwachen Haushalten zu Wohnraum in angespannten Wohnungsmärkten die Möglichkeiten der Vermieter beschränken, in einem Großteil der Bestands- und Neuvermietungsverhältnisse die Mieten zu erhöhen. Bestimmungen des Landes, welche die schon durch öffentliche Interessen überformte Vertragsfreiheit im BGB im zentralen Element der Gegenleistung in Form der Höhe des Mietzinses radikal einschränken, als „das mietrechtliche Schuldverhältnis unberührt“ lassend zu qualifizieren, erscheint vor diesem Hintergrund wenig überzeugend. ${ }^{154}$ So wird etwa in bestehende Mietverhältnisse schon dadurch eingegriffen, dass dem Vermieter sein Anspruch auf Zustimmung des Mieters zu angemessenen Mieterhöhungen gemäß $§ 558$ Abs. 1 S. 1 BGB entzogen wird. Damit bleibt es dabei, dass die scharfen Berliner Moratoriums- und Absenkungsregelungen die ausdifferenzierten Vorschriften des BGB auf Bundesebene aushöhlen, da sie den Bundesregelungen letztlich innerhalb des weiten Anwendungsbereichs der Berliner Regelungen keinen relevanten Anwendungsraum mehr belassen und diese auch in der Sache konterkarieren. Denn der in den Bundesregelungen normierte Ausgleich zwischen den Mieterund Vermieterinteressen in angespannten Wohnungsmärkten wird radikal überformt. Auch das zentrale Ziel, bezahlbaren Wohnraum zu schaffen und einkommensschwachen Haushalten den Zugang zu Wohnungen in diesen Lagen weiterhin zu ermöglichen, wird aufgrund der Anpassungen im BGB inzwischen von diesem genauso verfolgt wie vom Berliner Regelungswerk.

\section{bb) Große Rechtsunsicherheit durch Berliner Regelung}

Erneut ist hervorzuheben, dass die in unterschiedlichem Maße zusätzliche Unterfütterung der Berliner Regelungen durch öffentlich-rechtliche Interventionsmöglichkeiten, die ohnehin im Gesetzgebungsverfahren deutlich abgeschwächt wurden, die Bewertung nicht ändert (dazu bereits oben 4.c) bb)). Dass überhöhte Zahlungen im BGB bereicherungsrechtlich zurückabgewickelt und dabei gegebenenfalls auch (vor Zivilgerichten) gerichtlich durchgesetzt werden müssen,

153 Fischer-Lescano/Gutmann/Schmid Landeskompetenzen für Maßnahmen der Mietpreisregulierung, Rechtsgutachten für die Rosa Luxemburg Stiftung, 2019, S. 16, abrufbar im WWW unter der URL https://www.rosalux.de/fileadmin/rls_uploads/pdfs/Studien/Studien_8-19_Mietpreisre gulierung_web.pdf (zuletzt abgerufen am 1.7.2020).

154 Dagegen zutreffend Herrlein/Tuschl NZM 2020, 218 (230). 
begründet dabei keine Grundverschiedenheit zur Berliner Regelung hinsichtlich der angeblich großen Rechtsunsicherheit mit Blick auf das BGB und der vermeintlich einfacheren öffentlich-rechtlichen Durchsetzbarkeit der Berliner Regelung. ${ }^{155}$ Vielmehr dürfte eher das Gegenteil der Fall sein: So handelt es sich bei der BGB-Regelung immerhin um eine solche, die in ihren Grundzügen bereits etabliert und auch in der letzten größeren Verschärfung durch die „Mietpreisbremse“ verfassungsgerichtlich bestätigt worden ist. Die Berliner Regelung ist nicht nur in vielfältiger Hinsicht verfassungsrechtlichen Risiken von der Kompetenzwidrigkeit über die Verletzung des Vertrauensschutzes bis hin zur Verletzung der Eigentumsfreiheit ausgesetzt. Aufgrund der in erheblichem Umfang vorgesehenen unbestimmten Regelungen - etwa zur Härtefallklausel - sind Rechtsstreitigkeiten und damit eine entsprechende Rechtsunsicherheit vorprogrammiert. Diese führen nicht nur zu erheblichen Unsicherheiten auf Seiten der Vermieter, sondern auch der Mieter. ${ }^{156}$

\section{cc) Unklarheiten hinsichtlich der Vertragsregelung unter dem kumulativen Regime von BGB und Berliner Regelung}

Hinzu kommen eine Reihe handwerklicher Mängel der Berliner Regelung und Schwierigkeiten im Zusammenspiel von BGB und Berliner Regelung, die die Anwendung des Gesetzes erschweren: So ist unklar, wie bei einer Wiedervermietung vorzugehen ist. Wenn Wohnraum ab dem 23. Februar 2020 wiedervermietet wird, ist es per Gesetz verboten, eine höhere als die „eingefrorene“ Miete zu verlangen. Liegt die „eingefrorene“ Miete allerdings oberhalb der für die Wohnung maßgeblichen Mietobergrenze (Mietentabelle), darf die Vermietung nur zur Mietobergrenze erfolgen. Bei der Mietobergrenze ist gegebenenfalls der Zuschlag für moderne Ausstattung und Modernisierung zu berücksichtigen. Bei Wohnungen, deren Vormiete geringer als 5,02 €/ $\mathrm{m}^{2}$ war, darf die Miete bei der Wiedervermietung um max. $1 €$ auf bis zu 5,02 $€ / \mathrm{m}^{2}$ erhöht werden, wenn eine moderne Ausstattung vorhanden ist. Hierdurch ist völlig unklar, welche Miete in der Praxis z. B.

155 So aber Fischer-Lescano/Gutmann/Schmid Landeskompetenzen für Maßnahmen der Mietpreisregulierung, Rechtsgutachten für die Rosa Luxemburg Stiftung, 2019, S. 19 und passim, abrufbar im WWW unter der URL https://www.rosalux.de/fileadmin/rls_uploads/pdfs/Studien/ Studien_8-19_Mietpreisregulierung_web.pdf (zuletzt abgerufen am 1.7.2020).

156 Siehe dazu eindringlich Knauthe Der Mietendeckel - Eine verfassungsrechtliche Analyse unter Berücksichtigung des Referentenentwurfs der Senatsverwaltung für Stadtentwicklung und Wohnen für das Gesetz zur Mietenbegrenzung im Wohnungswesen in Berlin (Berliner MietenWoG), gif Policy paper 3/2019, Oktober 2019, S. 18, abrufbar im WWW unter der URL https://www. gif-ev.de/onlineshop/download/direct,454 (zuletzt abgerufen am 1.7.2020). 
bei Neuvermietungen vereinbart werden darf, wenn man hypothetisch unterstellt, dass das Gesetz verfassungsgemäß wäre. Darf nunmehr eine BGB-Miete vereinbart werden, die höher ist als die Mietentabelle, und lediglich in Höhe der Mietentabelle gefordert werden und nach Auslaufen des „Mietendeckels“ gilt die BGB-Miete oder ist eine solche Vereinbarung gemäß § 134 BGB nichtig und es darf vertraglich nur die Miete gemäß „Mietendeckel“ vereinbart werden? Die landeseigene (!) Berlinovo unterstellt in ihren Annoncen gegenwärtig eine „vorläufige Miete“, die nach Auslaufen des Gesetzes auf die nach dem BGB zulässige Miete angehoben wird.

Unklar ist aber, ob eine solche Vereinbarung nicht nichtig ist gemäß § 134 BGB. ${ }^{157}$ Allerdings sagen die Gesetzesbegründung und das Gesetz nicht explizit, dass nur die Miete nach Mietentabelle vereinbart werden darf, sondern es darf nur diese gefordert werden. Dies ist nur ein Beispiel für die zahlreichen Auslegungsschwierigkeiten und -unsicherheiten im Falle der Anwendung des Gesetzes.

\section{dd) Jeweils Regelungen für einen begrenzten, aber leicht abweichenden Zeitraum}

Irritierend ist in diesem Zusammenhang auch das Argument, der gänzlich unterschiedliche Ansatz ergäbe sich daraus, dass die Berliner Regelung zeitlich auf fünf Jahre beschränkt sei. ${ }^{158}$ Auch dies entspricht exakt der Regelung der Kappungsgrenze nach $\S 558$ Abs. 3 S. 3 BGB, die für die erforderlichen Gebietsfestsetzungen durch Landesverordnungen einen Zeitraum von „höchstens fünf Jahren“ vorsieht, auch wenn diese Regelung im Gesetz - genauso wie die Berliner Regelung - verlängert werden kann.

\section{ee) Unterminierung der Vergleichsmiete nach BGB im Falle der Wirkung der Berliner Regelung}

Schließlich entsteht durch die Berliner Regelung auch eine weitere substantiell konterkarierende Wirkung für die Bundesregelung: Für Bestands- und auch für Neuvermietungen rekurriert die jeweilige Bundesregelung auf einen Vergleichs-

157 Siehe dazu auch den in Fn. 25 zitierten Beschluss der 67. Zivilkammer des LG Berlins, die insoweit offensichtlich eine abweichende Einschätzung zum AG Charlottenburg, Urt. v. 4. 3. 2020 213 C 136/19, Tz. 15 - juris, vertritt.

158 Fischer-Lescano/Gutmann/Schmid Landeskompetenzen für Maßnahmen der Mietpreisregulierung, Rechtsgutachten für die Rosa Luxemburg Stiftung, 2019, S. 16 und 18, abrufbar im WWW unter der URL https://www.rosalux.de/fileadmin/rls_uploads/pdfs/Studien/Studien_8-19_Miet preisregulierung_web.pdf (zuletzt abgerufen am 1.7.2020). 
mietenansatz, der über den Mietenspiegel gerade auch durch Marktmieten geprägt wird. Dieser für das Erzielen der Regelungswirkung des BGB (Orientierung an Vergleichsmieten mit begrenzter Überformung durch Kappungsgrenzen) erforderliche Referenzwert entfällt aber künftig, da der Referenzmaßstab der Vergleichsmieten nicht mehr besteht bzw. im Wesentlichen ausgehöhlt wird. Als im Markt gebildete Vergleichsmieten stehen nur noch die wohl knapp 10\% der Mietverhältnisse zur Verfügung, die sich auf die Vermietung von Objekten beziehen, die nicht in den Anwendungsbereich der Berliner Regelung fallen, insbesondere weil die Gebäude erst nach dem Stichtag des 1. Januar 2014 (§ 1 Nr. 3 MietenWoG Bln) errichtet wurden. Damit wird letztlich das gesamte Vergleichsmietenmodell, das der Bundesregelung zugrunde liegt, unterlaufen, da keine dynamischen Vergleichsmieten mehr bestehen, sondern, wenn die Berliner Regelung ihre volle Wirkung entfaltet, im Wesentlichen nur noch Mieten gemäß der gesetzlich festgelegten, weitgehend statischen Mietentabelle in $\S 6$ MietenWoG Bln.

\section{ff) Analyse der Wirkungsrichtungen belegt Widersprüchlichkeit}

Daher ist es auch nicht möglich, die Frage nach der Widersprüchlichkeit der Regelungen zu beantworten, ohne eine Analyse ihrer auf das gleiche Marktsegment gerichteten, aber konträren Regelungswirkung unter einer verständigen Würdigung des gesamten Regelungsmodells im sozialen Mietrecht des BGB vorzunehmen. Erst daraus erschließt sich, dass es keineswegs „fernliegend“159, sondern wenn nicht zwingend, so doch zumindest äußerst zweckmäßig ist, dass der Bundesgesetzgeber zur Mietpreishöhe eine abschließende Regelung der Gestaltungsbefugnis der Länder im Rahmen der abschließenden Festlegung der Reichweite der Verordnungsermächtigungen vornimmt. Dass er dabei auch unter Beachtung der Treuepflichten im Bund-Länder-Verhältnis im Rahmen der Wahrnehmung seiner konkurrierenden Gesetzgebungskompetenz für die Frage der Prüfung, ob angespannte Wohnungsmärkte vorliegen, (und für die Absenkung der Kappungsgrenze um 5 Prozentpunkte von 20\% auf 15\%) die insoweit tatsächlich „sachnäheren Länder“ ermächtigt, ist ebenfalls vernünftig. Dass er schließlich die Fragen, ob ein Vergleichsmietenmodell oder ein Modell mit hoheitlich fixierten Tabellenmieten, ob also eine marktnahe oder eine marktferne Fixierung der Mieten und demnach eine fast vollständige Entkoppelung von

159 So aber Kingreen Zur Gesetzgebungskompetenz der Länder für das Öffentliche Mietpreisrecht bei Wohnraum, 2020, Kurzgutachten für die Bundestagsfraktion DIE LINKE., S. 16, abrufbar im WWW unter der URL https://www.linksfraktion.de/fileadmin/user_upload/PDF_Dokumente/ 2020/200218_Kingreen_Mietendeckel_Kompetenz.pdf (zuletzt abgerufen am 1.7.2020). 
Marktmieten erfolgen soll, nicht den Ländern überlassen möchte, ist ebenfalls sehr vernünftig, da die Länder andernfalls das austarierte System der Mietenregulierung im BGB sprengen könnten, wie der Berliner Regelungsversuch beweist.

\section{Kein zulässiges Gebrauchmachen einer Länderkompetenz für das „Wohnungswesen“ in Kombination mit der Rücksichtnahmepflicht des Bundes}

Schließlich ist ergänzend darauf hinzuweisen, dass auch keine Länderkompetenz für das „Wohnungswesen“ in Kombination mit einer Rücksichtnahmepflicht des Bundes auf die Länderkompetenz eine Regelungskompetenz der Länder für eine Mietpreisregulierung schafft, die dem erkennbaren Gestaltungswillen des Bundesgesetzgebers zu Mietpreisanpassungen widerspricht.

So wird teilweise in der Literatur vertreten, dass sich der Bundesgesetzgeber, da eine Kompetenzgemengelage zwischen der konkurrierenden Kompetenz des Bundes zur Mietpreisregelung aus Art. 74 Abs. 1 Nr. 1 GG und der residualen Länderkompetenz für das „Wohnungswesen“ aus Art. 70 GG vorliege, besonders kompetenzschonend zu verhalten habe und keine umfassende Sperrwirkung der Miethöhenregulierung gegenüber einer verschärften bzw. konterkarierenden Miethöhenregulierung der Länder erlassen dürfe. ${ }^{160}$ Dagegen spricht schon, dass der Bund gegen die Bundestreue erst dann verstößt, wenn er seine Kompetenz missbräuchlich in Anspruch nimmt. ${ }^{161}$ Ein solcher Missbrauch liegt nicht vor, da der Bund vor dem Land Berlin tätig geworden ist und dem Landesgesetzgeber im Bereich des Wohnungswesens noch eine Vielzahl von Maßnahmen (außerhalb eines Mietpreisrechts ausgerechnet für das Marktsegment der frei am Wohnungsmarkt verfügbaren Mietwohnungen) möglich bleiben.

160 So insbesondere Fischer-Lescano/Gutmann/Schmid Landeskompetenzen für Maßnahmen der Mietpreisregulierung, Rechtsgutachten für die Rosa Luxemburg Stiftung, 2019, S. 12f. und passim, abrufbar im WWW unter der URL https://www.rosalux.de/fileadmin/rls_uploads/pdfs/ Studien/Studien_8-19_Mietpreisregulierung_web.pdf (zuletzt abgerufen am 1.7.2020).

161 BVerfGE 4, 115, Rn. 72f. - juris, spricht von einer „Kontrolle der Einhaltung äußerster Grenzen“; BVerfGE 81, 310, Rn. 98 - juris, für die Verwaltungstätigkeit des Bundes; Herbst Gesetzgebungskompetenzen im Bundesstaat, 2014, S. 288; Wagner Die Konkurrenzen der Gesetzgebungskompetenzen von Bund und Ländern, 2011, S. 263f. m.w. N. aus der Rechtsprechung des BVerfG. 
Von der Gegenansicht wird insbesondere auf die bekannte Entscheidung des BVerfG zur kommunalen Verpackungssteuer verwiesen, ${ }^{162}$ deren lenkende Wirkungen in Widerspruch zu den Vorgaben in der vom Bundesgesetzgeber geregelten Abfallwirtschaft standen. Dabei wird insbesondere die Feststellung des BVerfG ins Feld geführt, dass im Falle eines Widerspruchs nicht stets dem Bundesrecht Vorrang zukomme. ${ }^{163}$ Nachdem das BVerfG die „Verpflichtung zur Beachtung der bundesstaatlichen Kompetenzgrenzen und zur Ausübung der Kompetenz in wechselseitiger bundesstaatlicher Rücksichtnahme“ nicht nur im Grundsatz der Bundestreue, sondern auch im Rechtsstaatsprinzip verortet und daraus den Grundsatz der Widerspruchsfreiheit der Rechtsordnung abgeleitet hat ${ }^{164}$, stellte das Gericht fest, dass ,grundsätzlich nach dem Rang, der Zeitenfolge und der Spezialität der Regelungen“165 darüber zu entscheiden sei, welche Regelung im Konfliktfall zu weichen habe. Auch nach diesem Maßstab setzt sich aber die Bundesregelung als vorrangig durch. Die Regelungen im BGB sind als formelle Gesetze des Bundes zunächst ranghöher als ein Landesgesetz, wobei dies im Falle der Annahme einer - hier abgelehnten - Kompetenz der Länder aus Art. 70 GG angesichts der prinzipiellen Gleichrangigkeit der Kompetenzen des Bundes aus Art. 74 GG und der Länder aus Art. 70 GG $^{166}$ in der weiteren Entwicklung der Rechtsprechung des BVerfG an sich keine größere Rolle spielen dürfte.

Auch die, daher vorliegend wichtigere, Zeitenfolge spricht für einen Vorrang der Bundesregelung. Denn diese ist zum einen deutlich früher als die Berliner Regelung erlassen worden. ${ }^{167}$ Zum anderen wurde die Bundesregelung auch

162 Siehe den Hinweis bei Fischer-Lescano/Gutmann/Schmid Landeskompetenzen für Maßnahmen der Mietpreisregulierung, Rechtsgutachten für die Rosa Luxemburg Stiftung, 2019, S. 13, abrufbar im WWW unter der URL https://www.rosalux.de/fileadmin/rls_uploads/pdfs/Studien/ Studien_8-19_Mietpreisregulierung_web.pdf (zuletzt abgerufen am 1.7.2020) auf BVerfGE 98, 106, Rn. 58 - juris.

163 Fischer-Lescano/Gutmann/Schmid aaO, S. 13.

164 BVerfGE 98, 106, Rn. 58 - juris: „Das Rechtsstaatsprinzip verpflichtet alle rechtsetzenden Organe des Bundes und der Länder, die Regelungen jeweils so aufeinander abzustimmen, dass den Normadressaten nicht gegenläufige Regelungen erreichen, die die Rechtsordnung widersprüchlich machen.“.

165 BVerfGE 98, 106, Rn. 58 - juris.

166 So konsequent Kingreen Zur Gesetzgebungskompetenz der Länder für das Öffentliche Mietpreisrecht bei Wohnraum, 2020, Kurzgutachten für die Bundestagsfraktion DIE LINKE., S. 20 ff., abrufbar im WWW unter der URL https://www.linksfraktion.de/fileadmin/user_upload/ PDF_Dokumente/2020/200218_Kingreen_Mietendeckel_Kompetenz.pdf (zuletzt abgerufen am 1.7.2020).

167 Die Kappungsgrenze des $\S 558$ Abs. 3 BGB enthält die Absenkungsmöglichkeit der Landesregierungen seit dem 1.5.2013, die „Mietpreisbremse“ des §556d BGB trat am 1.6.2015 in Kraft. 
zeitlich nach Bekanntwerden und Diskussion der Berliner Bestrebung vom Bundestag durch die Verlängerung und Verschärfung der „Mietpreisbremse“ nochmals bestätigt. Verschärfende Schritte im Sinne der Berliner Regelung wurden vom Bundestag aber gerade abgelehnt. ${ }^{168}$

Am stärksten wiegt schließlich, dass die Bundesregelung die speziellere Regelung ist, da diese für die Miethöhen auf der Basis einer Vergleichsmiete eine spezifische Höhenregelung trifft und dabei bereits zum sozialen Ausgleich beiträgt. Die Länder müssen zur Ausschöpfung des Kompetenztitels des Wohnungswesens und zur Schaffung einer spezielleren Regelung eine davon abweichende, sachmateriell eigenständige Regelung treffen, indem etwa ein eigenes Marktsegment reguliert wird oder eine unterstützende bzw. ergänzende Regelung für dasselbe Marktsegment getroffen wird. Die Absenkung einer zulässigen Mieterhöhung von 15\% auf 0\% im selben Marktsegment stellt jedoch nicht die Wahrnehmung einer eigenen, spezielleren Gestaltungsmöglichkeit dar, sondern regelt den identischen Gegenstand mit denselben Motiven lediglich abweichend im Sinne einer radikalen Verschärfung. Denkbar wären insoweit etwa Mietpreisbindungen im öffentlich geförderten Wohnraum oder im Rahmen einer Wohnraumbewirtschaftung, die aufgrund einer (vorliegend allerdings nicht gegebenen) akuten Wohnungsnot die freie Verfügungsgewalt über das Mietobjekt etwa durch Belegungsrechte entzieht. Für das Marktsegment der frei am Wohnungsmarkt angebotenen Mietwohnungen kann dagegen kein gegenüber dem BGB abweichendes (vorliegend verschärfendes, aber auch kein großzügigeres) Mietpreisrecht von den Ländern erlassen werden. ${ }^{169}$

168 Siehe die Ausführungen oben bei den Fn. 58-61.

169 Wissenschaftlicher Dienst des Deutschen Bundestages, Gesetzliche Mietpreisregulierung durch die Länder aufgrund der Gesetzgebungskompetenz für das Wohnungswesen, WD 3 3000-029/19 vom 5.2.2019, S. 6. 